\title{
Nonlinear run-ups of regular waves on sloping structures
}

\author{
T.-W. Hsu ${ }^{1,}$, S.-J. Liang ${ }^{2}$, B.-D. Young ${ }^{1}$, and S.-H. Ou ${ }^{1}$ \\ ${ }^{1}$ Department of Hydraulic and Ocean Engineering, National Cheng Kung University, Tainan 701, Taiwan \\ ${ }^{2}$ Department of Marine Environmental Informatics, National Taiwan Ocean University, Keelung 202, Taiwan \\ *now at: Research Center for Ocean Energy and Strategies, National Taiwan Ocean University, Keelung 202, Taiwan
}

Correspondence to: T.-W. Hsu (twhsu@ mail.ncku.edu.tw)

Received: 22 June 2012 - Revised: 11 November 2012 - Accepted: 15 November 2012 - Published: 21 December 2012

\begin{abstract}
For coastal risk mapping, it is extremely important to accurately predict wave run-ups since they influence overtopping calculations; however, nonlinear run-ups of regular waves on sloping structures are still not accurately modeled. We report the development of a high-order numerical model for regular waves based on the second-order nonlinear Boussinesq equations (BEs) derived by Wei et al. (1995). We calculated 160 cases of wave run-ups of nonlinear regular waves over various slope structures. Laboratory experiments were conducted in a wave flume for regular waves propagating over three plane slopes: $\tan \alpha=1 / 5,1 / 4$, and $1 / 3$. The numerical results, laboratory observations, as well as previous datasets were in good agreement. We have also proposed an empirical formula of the relative run-up in terms of two parameters: the Iribarren number $\xi$ and sloping structures $\tan \alpha$. The prediction capability of the proposed formula was tested using previous data covering the range $\xi \leq 3$ and $1 / 5 \leq \tan \alpha \leq 1 / 2$ and found to be acceptable. Our study serves as a stepping stone to investigate run-up predictions for irregular waves and more complex geometries of coastal structures.
\end{abstract}

\section{Introduction}

As a wave propagates toward relatively shallow water prior to breaking, a part of its energy is dissipated on the slope of shore structures or on beaches. The wave run-up refers to the maximum vertical extent of a wave up-rush on a beach or structure above still water levels. It is extremely important to accurately predict a wave run-up in order to determine the required crest elevations for a sloping coastal structures. For coastal risk mapping, a good estimate of the wave runup is valuable since it is closely related to the calculation of overtopping; moreover, wave run-up data may also be used for estimating overtopping or for delimiting a buffer zone to protect coastal infrastructure from extreme run-ups.

Based on the linear Lagrangian equation of motion for shallow water, Miche (1944) derived an equation for estimating wave run-ups: $R_{\mathrm{u}} / H_{0}=\sqrt{\pi / 2 \alpha}$, where $R_{\mathrm{u}}$ is the maximum run-up height; $H_{0}$, wave height in deep water; and $\alpha$, the angle of the slope. Nevertheless, it is difficult to obtain an accurate theoretical formula for the wave run-up height due to the wave nonlinearity and breaking near the shallow water region. Numerous researchers (e.g., Granthem, 1953; Saville, 1955, 1956, 1958; Savage, 1958; Toyoshima et al., 1964) have measured wave run-ups along various types of smooth and rough sloping, recurved, composite, and stepped structures. Notably, the run-up measurements for the above mentioned structures were based on regular waves in laboratory experiments. Table 1 summarizes some empirical formulae for regular wave run-ups; where, $\alpha_{\mathrm{c}}$ denotes the critical slope for the limiting condition of wave breaking; $K_{\mathrm{s}}$, the shoaling coefficient; $H$, the wave height at water depth $h$ at the foot of the slope; and $h_{0}$, the midpoint elevation of standing waves from still water levels. Miche's equation was modified by Takada (1970) based on experimental data, and is presented in Table 1. The formulae for breaking wave run-ups on smooth and rough plane slopes as well as composite slopes were proposed by Hunt (1959). For regular waves breaking on a sloping beach, the dimensionless equation for the maximum run-up $R_{\mathrm{u}}$ is given by $R_{\mathrm{u}} / H=\xi$; here $\xi$ is the Iribarren number defined as $\xi=\tan \alpha / \sqrt{H / L_{0}}$ (e.g., Battjes, 1974), where $L_{0}$ is the wavelength for the deep water condition. The design formula for estimation run-ups of regular waves is given in the Shore Protection Manual (SPM) (1984), in which some extensions have been made based on a reanalysis 
Table 1. Different empirical formulae for regular wave run-ups.

\begin{tabular}{|c|c|c|}
\hline Authors & Formulae & Wave breaking or non-breaking \\
\hline Miche (1944) & $\frac{R_{\mathrm{u}}}{H_{0}}=\sqrt{\frac{\pi}{2 \alpha}}$ & non-breaking \\
\hline \multirow{2}{*}{ Hunt (1959) } & $\frac{R_{\mathrm{u}}}{H_{0}}=3$ & surging breaker \\
\hline & $\frac{R}{H}=\xi$ & breaking on the slope \\
\hline Takada (1970) & $\begin{array}{l}\frac{R_{\mathrm{u}}}{H_{0}}=\left(\sqrt{\frac{\pi}{2 \alpha}}+\frac{h_{0}}{H}\right) K_{\mathrm{S}} \\
\text { for } \frac{H_{0}}{L_{0}} \leq \sqrt{\frac{2 \alpha}{\pi}} \frac{\sin ^{2} \alpha}{\pi} \\
\frac{R_{\mathrm{u}}}{H_{0}}=\left(\sqrt{\frac{\pi}{2 \alpha}}+\frac{h_{0}}{H}\right) K_{\mathrm{S}}\left(\frac{\cot \alpha_{\mathrm{c}}}{\cot \alpha}\right)^{2 / 3} \\
\text { for } \frac{H_{0}}{L_{0}}>\sqrt{\frac{2 \alpha}{\pi}} \frac{\sin ^{2} \alpha}{\pi}\end{array}$ & non-breaking, $\tan \alpha \leq 1 / 8$ \\
\hline Hughes (2004) & $\frac{R_{\mathrm{u}}}{h}=3.84 \tan \alpha \sqrt{\frac{M_{F}}{\rho g h^{2}}}$ & non-breaking \\
\hline Present Paper & $\begin{array}{l}\frac{R}{H}=\xi, \text { for } \xi \leq 2, \tan \alpha<1 / 5 \\
\frac{R_{\mathrm{u}}}{H}=2\left(\frac{\xi}{2}\right)^{0.04 / \tan ^{2} \alpha}, \text { for } \xi>2, \tan \alpha>1 / 5\end{array}$ & breaking on the slope \\
\hline
\end{tabular}

of Stoa (1978), which, in turn, is essentially the same form as Hunt (1959).

Hughes (2004) re-examined wave run-up data for regular, irregular, and solitary waves breaking on smooth impermeable plane slopes. He presented a new wave run-up equation by introducing a wave parameter representing the maximum depth-integrated wave momentum flux, as presented in Table 1. Key dimensionless parameters such as the relative wave height $H / h$, the relative wavelength $k h$ (where $k$ is the wavenumber), and sloping structures $\tan \alpha$, which affect the maximum vertical run-up elevation, are included in the equation. Hsiao et al. (2008) presented laboratory experimental data from a large wave flume of breaking solitary waves. They used the experimental data to re-examine existing formulae and proposed a simple formula to predict the maximum wave run-up height on a uniform beach with the slope ranging from $1 / 15$ to $1 / 60$.

The abovementioned empirical formulae provide useful information for practical applications, but they are generally limited to a relatively small number of data and to simplified sloping structures. Extrapolation may be required for practical use, but they may be invalid. For example, after several years of practical use, it appears that extreme situations like hurricanes or typhoons are almost never exactly the same as those predicted using these empirical formulae (TAW, 2002). Furthermore, run-up formulae require the representative wave height and period at the toe of the structures as the input, but when the toe is inside the surf zone, it is difficult to specify the representative wave height and period accurately.
An alternative method for describing wave run-ups on a beach or other structures is the development and application of numerical models that predict temporal and spatial variations of run-up elevations. A depth-averaged nonlinear shallow water equation (Kobayashi, 1989; Raubenheimer and Guza, 1996; Raubenheimer, 2002) is widely used to simulate wave run-ups in engineering practice. However, the model based on a shallow water equation is constricted in the range of long waves or waves propagating in a shallow water region, i.e., $h / L \leq 1 / 20$, where $L$ is the local wavelength at water depth $h$. In fact, the general form of Boussinesq equations (BEs) may be sufficient for modeling wave run-up processes over a wider range of water depth regions (from deep water depth, $h / L \geq 1 / 2$, to shallow water depth). For modeling wave breaking, Tao (1983) and Zelt (1991) incorporated the concept of artificial eddy viscosity into the momentum equation of BEs. However, the new term for wave breaking did not satisfy the principle of conservation of momentum; this problem was subsequently overcome by Kennedy et al. (2000). Moreover, it has been difficult to combine numerical treatments of wave run-ups - a time-dependent dry-wet moving interface with wave breaking. Tao (1984) advanced a solution by treating the seabed in the vicinity of a shoreline as a porous media or a narrow slot. Subsequently, Madsen et al. (1997) applied the method of Tao (1984) to BEs, whereas Kennedy et al. (2000) improved the solution and satisfied the principle of conservation of mass. For simulating wave run-ups, Lynett et al. (2002) and Nwogu and Demirbilek (2010) developed a numerical model that combined BEs with a dry-wet moving boundary 
technique. Fuhrman and Madsen (2008) as well as Madsen and Fuhrman (2008) employed so-called extrapolating boundary technique to handle the moving dry-wet interface with a high-order BEs. In addition to the surface elevation, the work established the shoreline velocity which could accurately simulate the moving dry-wet interface.

The wave run-up models discussed above are related to run-ups on weakly nonlinear and non-breaking waves. For handling the wave breaking and run-up problems, the current widely used BEs are limited primarily because of their weak nonlinearity (Hsu et al., 2004). Higher-order nonlinear and dispersive equations must be solved for wave breaking and run-ups on a steep structure using a higher-order numerical scheme.

The main purpose of this paper is to report the development and application of a higher-order numerical model based on the second-order nonlinear BEs derived by Wei et al. (1995) for regular waves. The model developed by Kennedy et al. (2000) was used to model the wave breaking and run-ups for regular waves. We also performed experiments on the run-ups associated with different sloping structures in a wave flume. The evolution of shoreline motions was recorded by using a wire that was installed above the sloping bottom. Laboratory observations were used to validate the numerical results calculated from the BEs for sloping structures varying from $1 / 5$ to $1 / 3$. This verification could confirm the usefulness of the second-order fully nonlinear BEs of Wei et al. (1995). Finally, numerical results obtained from a series of calculations for regular wave run-ups on a slope from a seawall were obtained, and empirical equations were then derived by regression analysis for practical applications. The equation was also validated by the datasets of Granthem (1953) and Saville (1955). The present paper provides limited but useful information that may be valuable and serve as a stepping stone to investigate run-up predictions for irregular wave run-ups on both plane and more complex sloping structures.

\section{Governing equations and numerical method}

Combining the 1-D, second-order, fully nonlinear BEs (Wei et al., 1995) and the modeling method for wave breaking and wave run-ups (Kennedy et al., 2000), the governing equations can be expressed as

$$
\begin{aligned}
\eta_{t} & =-E(\eta, u)=-E_{1}+f(x, t) \\
U_{t}^{*}(u) & =-F(\eta, u)=-F_{1}+R_{b x}+C_{1} u+C_{2} u_{x x},
\end{aligned}
$$

where

$$
\begin{aligned}
E_{1} & =\left\{A \left[u+\left(\left(h^{2} \beta^{2}\right) / 2-\left(h^{2}-h \eta+\eta^{2}\right) / 6\right) u_{x x}\right.\right. \\
& \left.\left.+(h \beta+(h-\eta) / 2)(h u)_{x x}\right]\right\}_{x} / b
\end{aligned}
$$

$$
\begin{aligned}
F_{1} & =g \eta_{x}+u u_{x}+\left\{2(h \beta-\eta) u(h u)_{x x}\right. \\
& +\left(h^{2} \beta^{2}-\eta^{2}\right) u u_{x x} \\
& \left.+\left[(h u)_{x}+\eta u_{x}\right]^{2}+2 \eta(h u)_{x t}+\eta^{2} u_{x t}\right\}_{x} / 2 \\
U^{*} & =u+\left[h^{2} \beta^{2} u_{x x}+2 h \beta(h u)_{x x}\right] / 2
\end{aligned}
$$

In Eqs. (3)-(5), $u$ is the horizontal velocity at an arbitrary depth; $\beta=z_{\alpha} / h$, where $z_{\alpha}$ is the vertical position of an arbitrary position; and $A$ and $b$, the area and width of the narrow slot, respectively, for modeling the wave run-up. The definitions of $A$ and $b$ are from Kennedy et al. (2000). In Eq. (2), the artificial eddy viscosity term $R_{b x}$ is given by

$R_{b x}=\left\{\frac{v_{t}[(h+\eta) u]_{x}}{(h+\eta)}\right\}_{x}$

$v_{t}$ is the dynamic eddy viscosity as a function of time and space, and it can be expressed as follows:

$v_{t}=B \delta_{b}^{2}(h+\eta)\left|\eta_{t}\right|$,

where $\delta_{b}$ is a non-dimensional parameter. A value of $\delta_{b}=1.2$ was proposed by Kennedy et al. (2000) based on the model calibration results. $B$ is assumed to vary steadily between 0 and 1 to avoid numerical instability for incipient breaking waves. BE model of Wei et al. (1995) is valid for $k h<3.8$, where $k$ is the wavenumber. The difference of phase speed between the present model and linear dispersion is less than $2 \%$ under $k h<\pi$.

In Eq. (1), $f(x, t)$ is the wave generating function (Wei and Kirby, 1995):

$$
\begin{aligned}
& f(x, t)= \\
& \frac{2 a \exp \left(k^{2} / 4 \beta_{1}\right)\left(\omega^{2}-\alpha_{1} g k^{4} h^{3}\right)}{\omega k \sqrt{\pi / \beta_{1}}\left[1-\alpha(k h)^{2}\right]} \times e^{-\beta_{1}\left(x-x_{0}\right)^{2}} \sin (\omega t+\varepsilon) .
\end{aligned}
$$

In Eq. (2), $C_{1} u$ and $C_{2} u_{x x}$ are the damping terms defined following Wei and Kirby's (1995) suggestion. The damping terms are applied to the "sponge layers" that are regions at both ends of the computational domain.

A finite difference scheme with staggered grids is used for the numerical computations. The first-order differential terms in the spatial domain have a fourth-order resolution aiming to reduce the effects of the down-shifting of the frequency in numerical calculations (Wei and Kirby, 1995), whereas the second- and third-order differential terms have second-order resolutions. For the computations of the temporal domain, the predictor-corrector method given by Wei and Kirby (1995) has been adopted. The predictor step applies the third-order explicit Adams-Bashforth method, whereas the corrector step uses the fourth-order implicit Adams-Moulton method. Details of the numerical scheme are given in Wei and Kirby (1995). 


\section{Laboratory experiments}

The experiments were carried out in a wave flume at the Department of Hydraulic and Ocean Engineering of National Cheng Kung University, Taiwan. The dimensions of the wave flume are $25 \mathrm{~m}$ long, $0.5 \mathrm{~m}$ wide, and $0.6 \mathrm{~m}$ deep, respectively. Target regular waves were generated at one end of the flume by using a piston-type wave maker. A plane beach with three different slopes $-1 / 3,1 / 4$, and $1 / 5$ (corresponding to $18.43^{\circ}, 14.04^{\circ}$, and $11.31^{\circ}$, respectively) - was built by using a smooth layer of a wooden model placed at $x=15 \mathrm{~m}$ starting from the wave board. A preliminary run without any structure was conducted to estimate the wave reflection and to establish the desired amplitude of the incident wave in the wave flume.

A schematic diagram of the experimental setup is shown in Fig. 1. The offshore wave conditions were $1.71 \mathrm{~cm} \leq$ $H_{0} \leq 7.26 \mathrm{~cm}$ and $0.8 \mathrm{~s} \leq T \leq 2.0 \mathrm{~s}$ for the wave height and wave period, respectively, which were determined from an automatic wave maker system and adjusted using the first wave gauge located at $x=9.9 \mathrm{~m}$ by subtracting the reflected waves due to the structure. The water depth was kept constant at $h=40 \mathrm{~cm}$, and the wave steepness was within the range of $\varepsilon=0.003 \sim 0.073$, where $\varepsilon=H_{0} / L_{0}$ is the wave steepness in deep water. Table 2 summarizes the laboratory experimental conditions and measured wave run-ups. The measurement apparatus was arranged and deployed with wave gauges and run-up wires, as illustrated in Fig. 1. In order to obtain a higher resolution of the local water surface elevation, six capacitance-type wave gauges were deployed at $7.6 \mathrm{~cm}$ intervals between $16.1 \mathrm{~m}<x<16.49 \mathrm{~m}$ downstream of the wave maker. The measured data were used to analyze wave breaking, wave profile decay, and run-up height. All the wave gauges were calibrated using a standard procedure in which the water level was changed to adjust the response voltage of each gauge to ensure its linearity and stability. The linear relationship of the gauge response was given by a linear coefficient of 0.99 , and this is consistent with the report of Hsu et al. (2002). Each wave gauge had a 16-bit digitization with noise less than $0.5 \mathrm{~mm}$. The duration of the shoreline motions was continuously recorded by a run-up wire that was installed above the sloping bottom, as shown in Fig. 1b. Note that the run-up wire was enclosed in a plastic tube fixed securely on the sloping bed.

The synchronization of signals from parallel inputs in the wave flume must be considered; hence, to cope with this problem, the data acquisitions of local water surface elevations were recorded simultaneously with a sampling rate of $100 \mathrm{~Hz}$ using the multi-nodes data acquisition system (Hsiao et al., 2008).
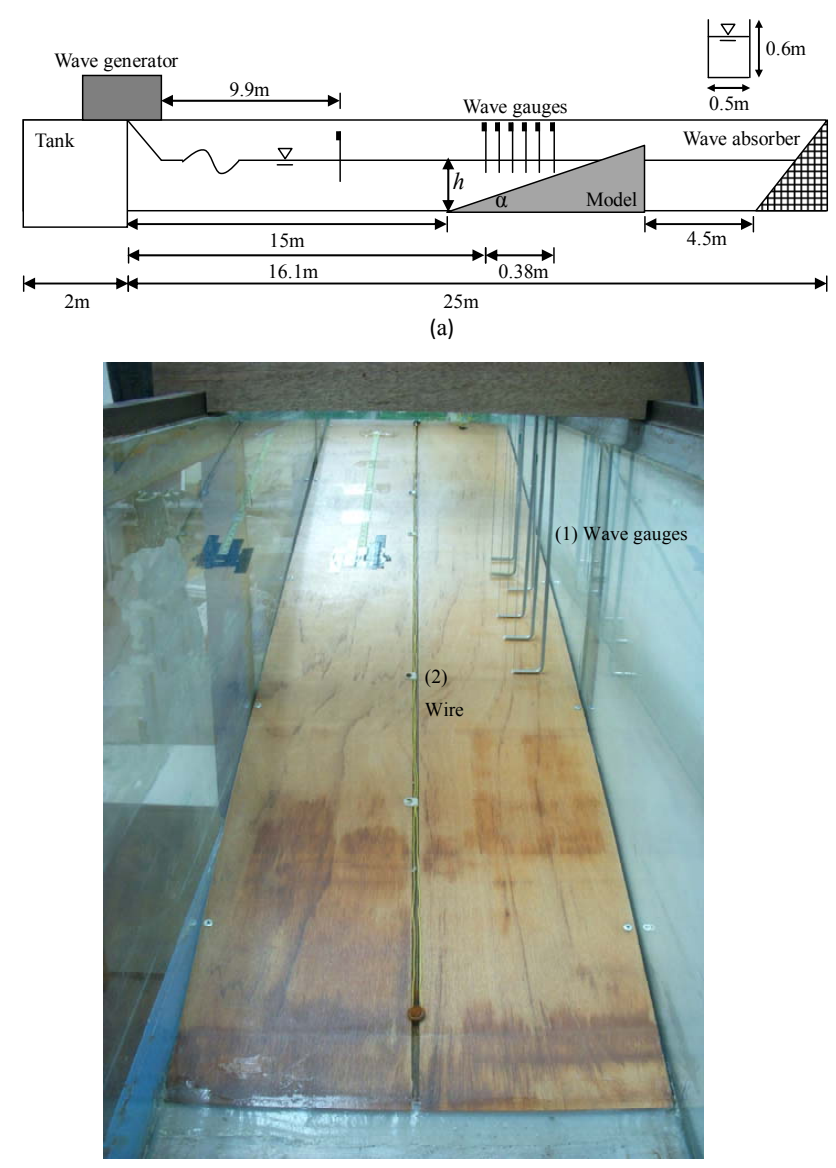

(b)

Fig. 1. Experimental setup: (a) wave flume and (b) wave gauges and a wire for measuring wave height and run-up.

\section{Model verification}

In this section, we verify the applicability of the present numerical model by comparing the computed results with various input wave conditions obtained from laboratory experiments. Specific attention is given to the spatial variations in wave height and wave run-up height on a slope under wavebreaking conditions. $\Delta x=L / 100$ and $\Delta t=T / 100$ are used for all computations, where $L$ is the local wavelength and $T$ is the wave period, respectively.

First, the numerical results (solid line in Fig. 2) for wave height variations (shoaling and breaking) on a 1/20 sloping structure are compared with the results of the mild-slope equations (MSE) (dashed line; Hsu et al., 2005) and experimental data of Nagayama (1983). In general, the numerical results obtained from the present model agree better with the experimental results than with the MSE, but there is a slight discrepancy for broken wave heights.

A solitary wave of a relative height $H_{0} / h=0.28$ propagating on a uniform slope of $1: 19.85$ was also modeled. Computed results were compared with experimental data 
Table 2. Laboratory experimental conditions of wave run-up.

\begin{tabular}{|c|c|c|c|c|c|c|c|c|}
\hline \multicolumn{3}{|c|}{$\tan \alpha=1 / 3$} & \multicolumn{3}{|c|}{$\tan \alpha=1 / 4$} & \multicolumn{3}{|c|}{$\tan \alpha=1 / 5$} \\
\hline $\begin{array}{l}H \\
(\mathrm{~cm})\end{array}$ & $\begin{array}{l}T \\
\text { (s) }\end{array}$ & $\begin{array}{l}R_{\mathrm{u}} \\
(\mathrm{cm})\end{array}$ & $\begin{array}{l}H \\
(\mathrm{~cm})\end{array}$ & $\begin{array}{l}T \\
(\mathrm{~s})\end{array}$ & $\begin{array}{l}R_{\mathrm{u}} \\
(\mathrm{cm})\end{array}$ & $\begin{array}{l}H \\
(\mathrm{~cm})\end{array}$ & $\begin{array}{l}T \\
\text { (s) }\end{array}$ & $\begin{array}{l}R_{\mathrm{u}} \\
(\mathrm{cm})\end{array}$ \\
\hline 1.81 & 2.00 & 4.73 & 2.15 & 2.00 & 6.24 & 1.71 & 2.00 & 4.13 \\
\hline 3.09 & 1.80 & 7.72 & 2.65 & 2.00 & 7.12 & 1.98 & 2.00 & 3.84 \\
\hline 4.85 & 1.40 & 9.71 & 3.65 & 2.00 & 9.21 & 2.71 & 2.00 & 3.29 \\
\hline 5.08 & 1.00 & 7.93 & 4.05 & 2.00 & 8.56 & 2.77 & 2.00 & 3.25 \\
\hline 4.45 & 0.80 & 5.52 & 4.73 & 1.80 & 10.40 & 3.02 & 1.80 & 2.69 \\
\hline 5.36 & 0.80 & 6.27 & 4.35 & 1.60 & 9.22 & 3.37 & 1.80 & 2.55 \\
\hline 7.26 & 0.80 & 8.79 & 6.10 & 1.40 & 10.21 & 3.59 & 1.80 & 2.47 \\
\hline- & - & - & 5.14 & 1.20 & 7.76 & 4.03 & 1.80 & 2.33 \\
\hline- & - & - & 5.66 & 1.10 & 4.21 & 5.24 & 1.60 & 1.75 \\
\hline- & - & - & 5.52 & 1.00 & 7.02 & 5.48 & 1.60 & 1.71 \\
\hline- & - & - & 6.39 & 1.00 & 7.72 & 3.99 & 1.40 & 1.70 \\
\hline- & - & - & - & - & - & 4.29 & 1.40 & 1.64 \\
\hline- & - & - & - & - & - & 3.60 & 1.20 & 1.51 \\
\hline- & - & - & - & - & - & 4.51 & 1.20 & 1.35 \\
\hline - & - & - & - & - & - & 3.79 & 1.10 & 1.35 \\
\hline- & - & - & - & - & - & 4.36 & 1.10 & 1.26 \\
\hline - & - & - & - & - & - & 5.13 & 1.10 & 1.16 \\
\hline- & - & - & - & - & - & 4.31 & 1.00 & 1.16 \\
\hline
\end{tabular}

Note: $h=40 \mathrm{~cm}$

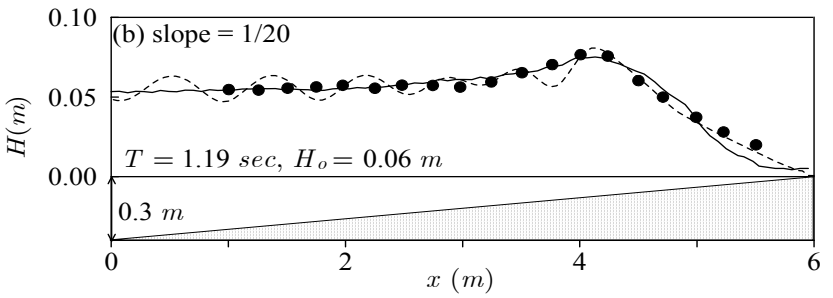

Fig. 2. Comparison of the results of wave height distribution along a uniform slope of 1:20 among the present numerical model (-), numerical computation using MSE (-, Hsu et al. 2005), and the laboratory experiments $(\bullet)$ of Nagayama (1983). Input wave conditions: $T=1.19 \mathrm{~s}, H_{0}=0.06 \mathrm{~m}$, and $h=0.3 \mathrm{~m}$ at the toe.

reported in Synolakis (1986). As shown in Fig. 3, the numerical results for the water surface elevation at four different time instances, $t(g / h)^{1 / 2}=15,20,25$, and 45 , respectively, agree well with the laboratory experiments. Lynett et al. (2002) did the same test with COULWAVE, and obtained good results as well. Therefore, the present numerical model is suitable for modeling wave run-up on a sloping seabed for wave breaking and run-up.

Figure 4 shows a comparison between the numerical results and measurements (the symbols for experimental data and numerical results are given in the inset). Note that the predicted wave run-up by the fully second-order BEs model agrees well with measured data for three sloping structures: $\tan \alpha=1 / 5,1 / 4$, and $1 / 3$. Therefore, it will be beneficial to modify the empirical formulae through regression analysis using the computed results of 160 cases and experimental data and propose an improved formula for practical applications.

\section{Results and discussions}

The key focus of the present paper is to investigate the wave run-up heights for regular waves breaking on sloping structures. A series of numerical calculations were carried out for regular waves propagating over various sloping structures with the aim of establishing empirical equations for predicting wave breaking and wave run-up heights. Wave conditions including 4 wave periods $(T=3.5,5,6.5$, and $8 \mathrm{~s})$, 16 wave steepnesses $\varepsilon(0.002,0.003,0.004,0.005,0.006$, $0.007,0.008,0.009,0.010,0.015,0.02,0.03,0.04,0.05$, $0.06,0.07)$, and 10 sloping structures $(\tan \alpha=1 / 10 \sim 1 / 1$ ), totaling 160 cases were simulated. The specified wave steepness includes wave nonlinearity and different types of wave breaking. The toe water depth $h$ was determined under the deep-water condition by $k_{0} h=\pi$.

Figure 5 presents the numerical results for different sloping structures. It is interesting to note that the run-up height is highly dependent on the slope of structures. For a given Iribarren number, the run-up height decreases as the slope angle increases. The regression equation of $R_{\mathrm{u}} / H=2(\xi / 2)^{m}$ is implemented in the analysis, and it is further expressed by the linear logarithmic regression: $\log \left(R_{\mathrm{u}} / H\right)=m \log (\xi / 2)+$ $\log 2$, where $m=F(\tan \alpha)$ is the gradient of the linear $\log$ arithmic regression. The coefficient $m$ is shifted slightly 

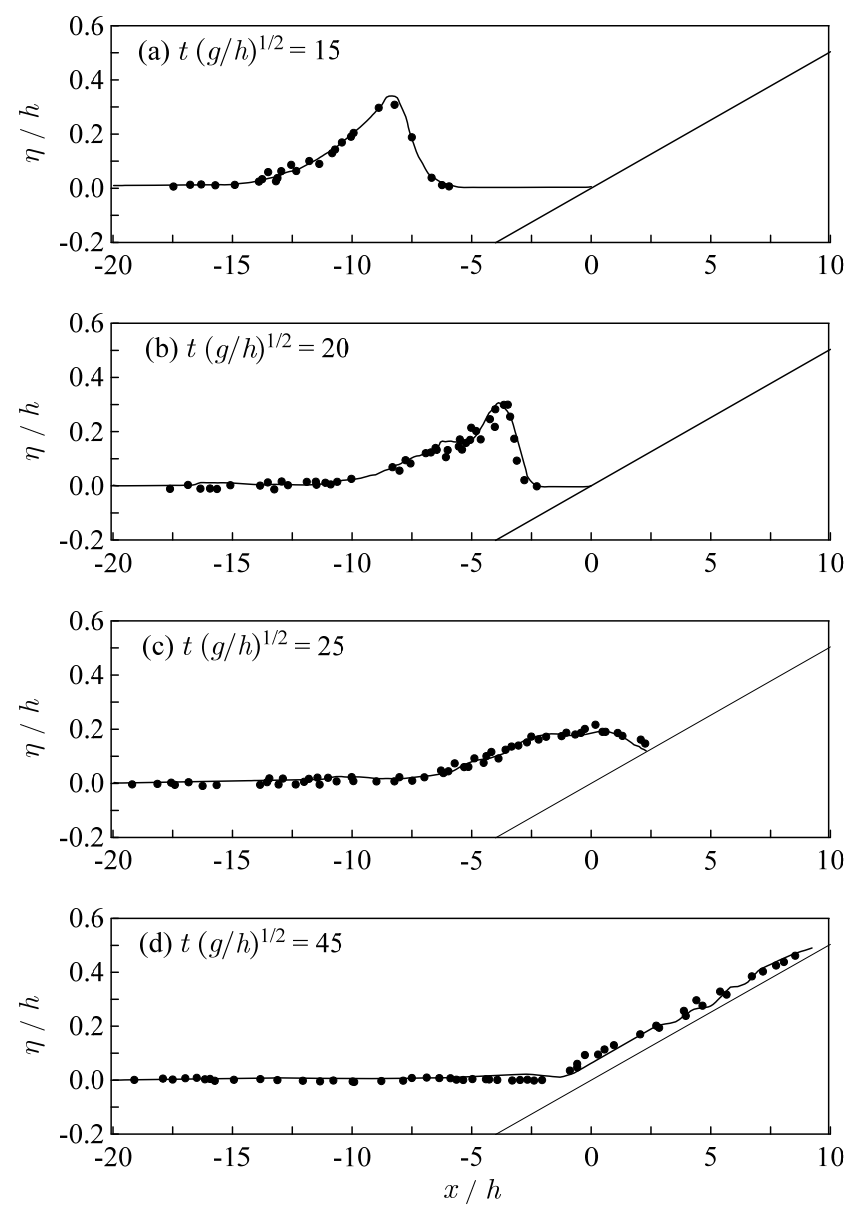

Fig. 3. Comparison of solitary wave propagating from breaking and run-up at four time instances between the present numerical model (-) and the laboratory experiments (•) of Synolakis (1986) on uniform slope of $1: 19.85$.

upward from the slope of structures. A more accurate equation $m=0.04 / \tan ^{2} \alpha$ is presented in Fig. 6. We notice that Fig. 6 clearly shows a close relationship between the dimensionless wave run-up $R_{\mathrm{u}} / H$ and the Iribarren number $\xi$ for different values of $m$. Figure 7 shows all datasets from numerical simulations and measured data of wave run-ups; herein, the measured run-up data for the rock-bubble structure of slope $\tan \alpha \leq 1 / 5$ were taken from Hunt (1959), and the numerical simulations of $\tan \alpha=1 / 2$ and $1 / 1$ were calculated from the BEs of Wei et al. (1995). The remaining are the laboratory data from the present experiments.

The result calculated using the wave run-up height equation $R_{\mathrm{u}} / H=\xi$ (Hunt, 1959) is also indicated (solid line for $\tan \alpha=1 / 5$ ). It is obvious that Hunt's equation overestimates the run-up height for $\xi>2$ and $\tan \alpha>1 / 5$. Notably, $R_{\mathrm{u}} / H$ increases linearly with $\xi$ for $\xi \leq 2$. However, the relationship of $R_{\mathrm{u}} / H$ and $\xi$ is very different for $\xi>2$ and $\tan \alpha>1 / 5$. Under these conditions, as the sloping angle increases, the wave run-up height decreases because of the increase in the

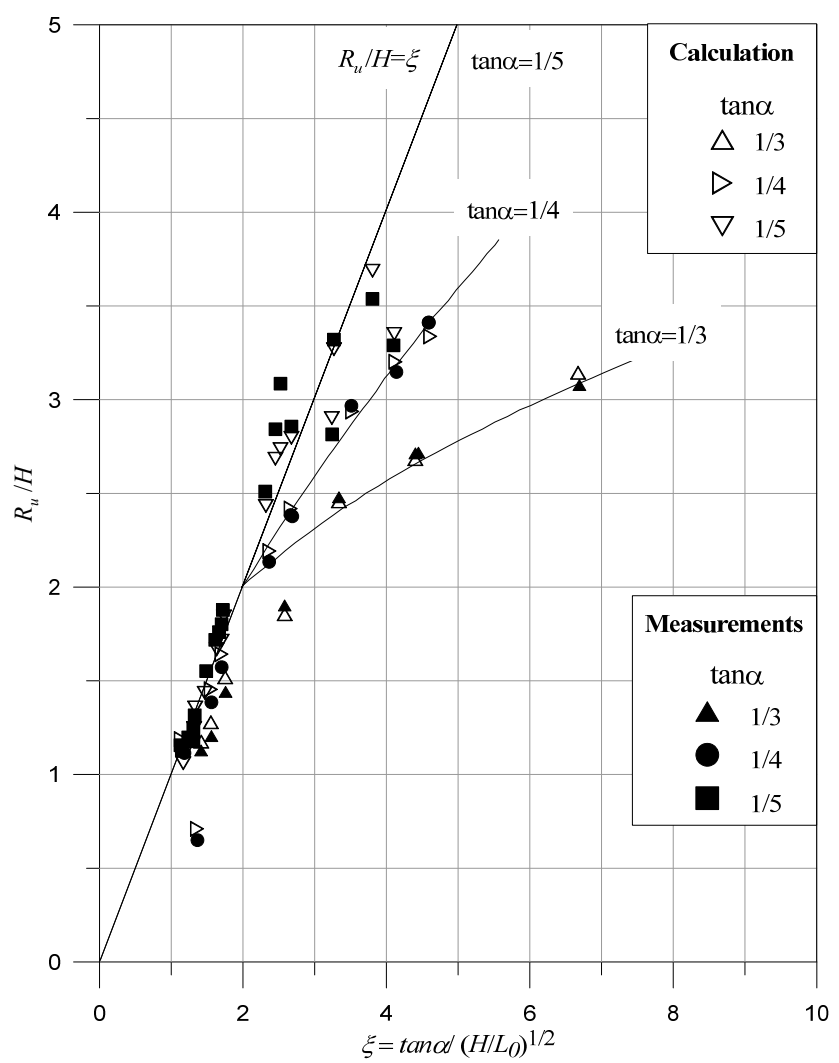

Fig. 4. Comparison on the run-up from numerical results with those from measurements.

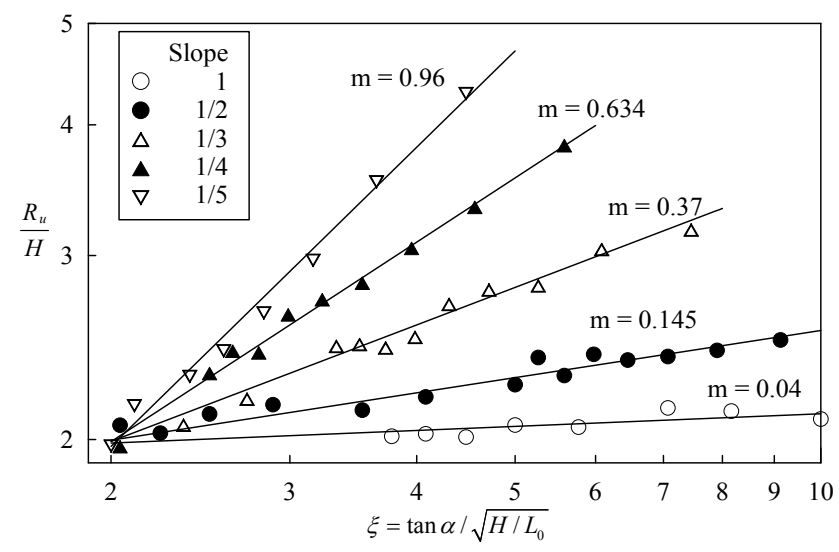

Fig. 5. Numerical results of $R_{\mathrm{u}} / H$ versus $\xi$ in logarithmic coordinate for different structure slopes.

downward swash from the fluid weight component $\rho g \sin \alpha$, where $\rho$ is the density of sea water and $g$ is the gravitational acceleration; this indicates that a steeper sloping structure produces a larger downward force that drags the water rushing upwards and results in a lower wave run-up height. The other reason may attribute to surging breakers in which the wave crest collapses and disappears. A considerable amount 


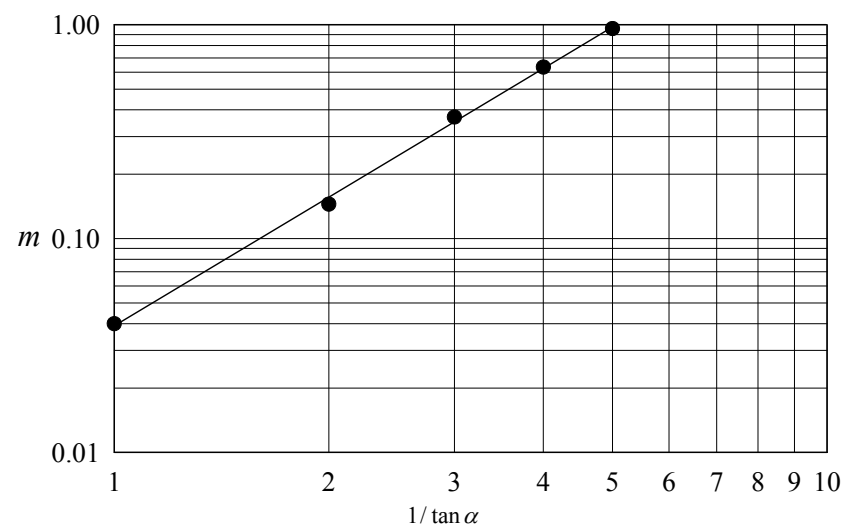

Fig. 6. The relation between the gradient $m$ and structure slope $\tan \alpha$.

of wave energy is released before the toe of the structure, and this results in reductions in the run-up height.

On close examination of the curves shown in Figs. 6 and 7 , we conjecture that these curves vary in a nonlinear power form with the slope angle parameter $\tan \alpha$ being the base parameter. In addition, Goda's (1975) experiment showed that wave-breaking characteristics are associated with the sloping angle when the waves reach the toe of the structure. Therefore, it is plausible to use the sloping angle as a parameter to distinguish the wave run-up formulae. Note that $R_{\mathrm{u}} / H=2$ for $\xi=2$ (Fig. 7) is a branch point of the wave run-up height for various sloping structures. Based on the analyses in Figs. 5 and 6, we performed a nonlinear regression analysis using the equation $\log \left(R_{\mathrm{u}} / H\right)=0.04 / \tan ^{2} \alpha \log (\xi / 2)+$ $\log 2$. The following empirical relationships for a wide range of $\xi$ are thus proposed:

$R_{\mathrm{u}} / H=\xi, \xi \leq 2$ or $\tan \alpha<1 / 5$

$R_{\mathrm{u}} / H=2(\xi / 2)^{0.04 / \tan ^{2} \alpha}, \xi>2$ and $\tan \alpha>1 / 5$

In Eq. (9), we use the surf similarity parameter to place the emphasis on the relative importance of wave breaking on a sloping beach. The beach slope is included in an independent parameter to identify the gravitational effects on the wave run-up on sloping structures. The method used in the present analysis appears to give a more realistic basis by focusing attention on the physical forces as separate terms in the appropriate equation of motion.

The results obtained using Eq. (9) are represented separately for the first four steep slopes $(\tan \alpha=1 / 1,1 / 2,1 / 3$, and $1 / 4$, respectively), as shown in Fig. 7. For $\tan \alpha<1 / 5$, we notice that Hunt's formula is still valid with the present derived equation. The correlation coefficient between the computed results from the empirical formula and the data is $R^{2}=0.99$ for $\tan \alpha \leq 1 / 5$, and $R^{2}=0.98$ for $\tan \alpha>1 / 5$. Kim and Lee (2009) indicated that Nwogu's (1993) model results were accurate up to $1: 1$ slope, but significantly inaccurate for steep slopes. The order of nonlinearity of our BE

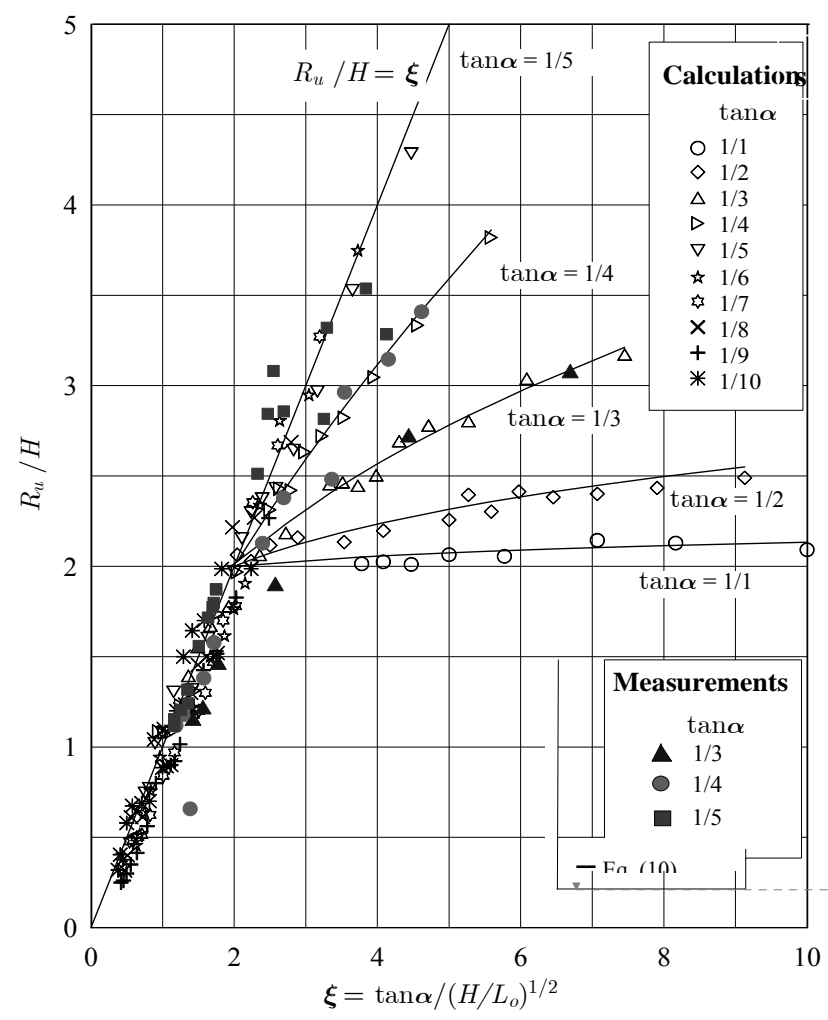

Fig. 7. Wave run-up height versus Iribarren number $\xi$ for regular wave transformation on various uniform slopes; the branch point at $\xi$ can also be seen.

model is higher than that of Nwogu's model. Prediction of our BE model should be reasonable for $1: 1$ slope simulation. Moreover, an agreement index $C_{R}$ proposed by Willmott (1981) is also used to evaluate the confidence of the regression equation, Eq. (10), which is defined as

$C_{R}=1-\frac{\sum_{i=1}^{N}\left(P_{i}-O_{i}\right)^{2}}{\sum_{i=1}^{N}\left[\left|P_{i}-\bar{O}\right|+\left|O_{i}-\bar{O}\right|\right]^{2}}$

where $P_{i}$ and $O_{i}$ denote predicted and observed data, respectively; $\bar{P}$ and $\bar{O}$, the mean values of $P_{i}$ and $O_{i}$, respectively; and $N$, the total number of evaluated points. The agreement index $C_{R}$ is approximately 0.99 . This result shows that the run-up for regular waves predicted by Eq. (10) shows an excellent agreement with the calculated data using BEs and measured data. Precisely defined statistical measures of the mean absolute error MAE $=|\bar{P}-\bar{O}|$, the root-mean-square error RMSE $=\sqrt{\frac{1}{N} \sum_{i=1}^{N}\left(P_{i}-O_{i}\right)^{2}}$, and the scatter index $\mathrm{SCI}=\mathrm{RMSE} / \bar{O}$ were also used to evaluate the regression agreement. The calculated results show that $\mathrm{MAE}=0.10, \mathrm{RMSE}=0.13$, and $\mathrm{SCI}=0.08$, which 


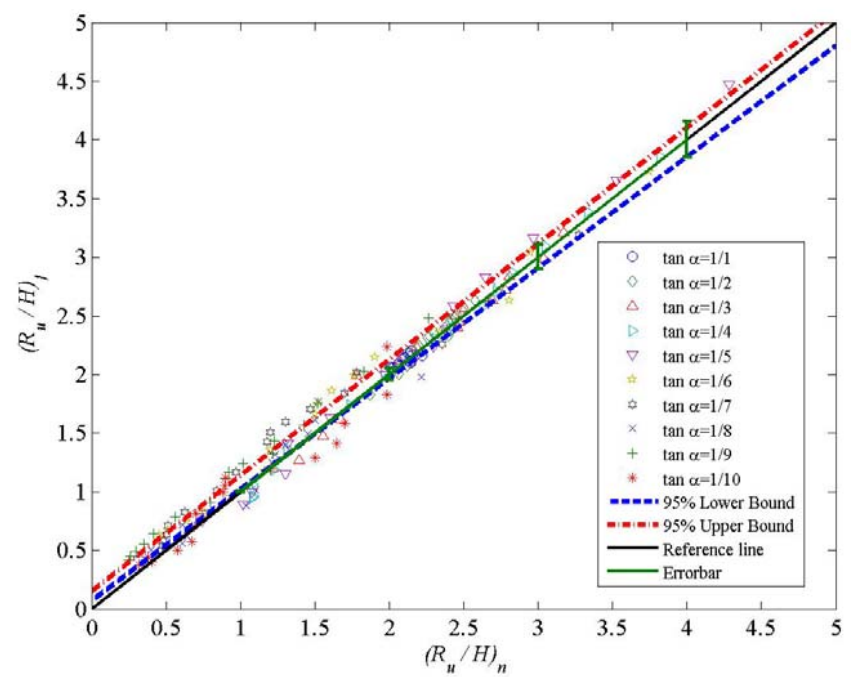

Fig. 8. Correlation and confidence interval between numerical model $\left(R_{u} / H\right)$ and empirical formula (Eq. 9).

indicates that Eq. (9) is able to offer good results for the calculated and observed data. Furthermore, good linear relationship can be found (Fig. 8) between the numerical results of the present model and those calculated from the empirical equations of Eq. (9). The confidence is in the range of $95 \%$ upper and lower bounds as shown in Fig. 8 .

Hughes (2004) re-examined existing wave run-up data for regular, irregular, and solitary waves on smooth and impermeable plane slopes. A model with a physical argument was used to derive a new wave run-up equation in terms of a wave parameter that represents the maximum momentum flux in a wave as it reaches the toe of the sloping structure. For regular waves, Granthem (1953) and Saville (1955) fitted the equation to regular wave run-ups for all the 152 datasets of laboratory tests in which the slopes ranging over $0.1 \leq \tan \alpha \leq 1$ were used in the reanalysis. The empirical formula is given in Table 1. Hughes' formula reproduces the data trend well, except for Granthem's results of $1: 1$ slope, which are lower than estimated. The same data have also been plotted in Fig. 9 using Hunt's (1959) formula. We note that the Iribarren number $\xi$ characterizes run-ups very well for $\xi \leq 2.0$. As slopes become steeper and $\xi>2.0$, the scatter increases. The data trend using linear regression seems to indicate that the branch point is around $\xi=2.5$. The formula $R / H=3$ was reported by Hunt (1959) for surging breaking, but it significantly overestimates the wave run-up height.

The proposed run-up relationship of Eq. (9) was further verified using laboratory run-up test results with the same data of Granthem (1953) and Saville (1955). Run-up values for different sloping structures were employed for validating the present formula. For comparison, the wave height at the toe of the structure was estimated by linear shoaling from the measured offshore wave height. The results are presented in Fig. 9. Note that most of the data follow the trend of

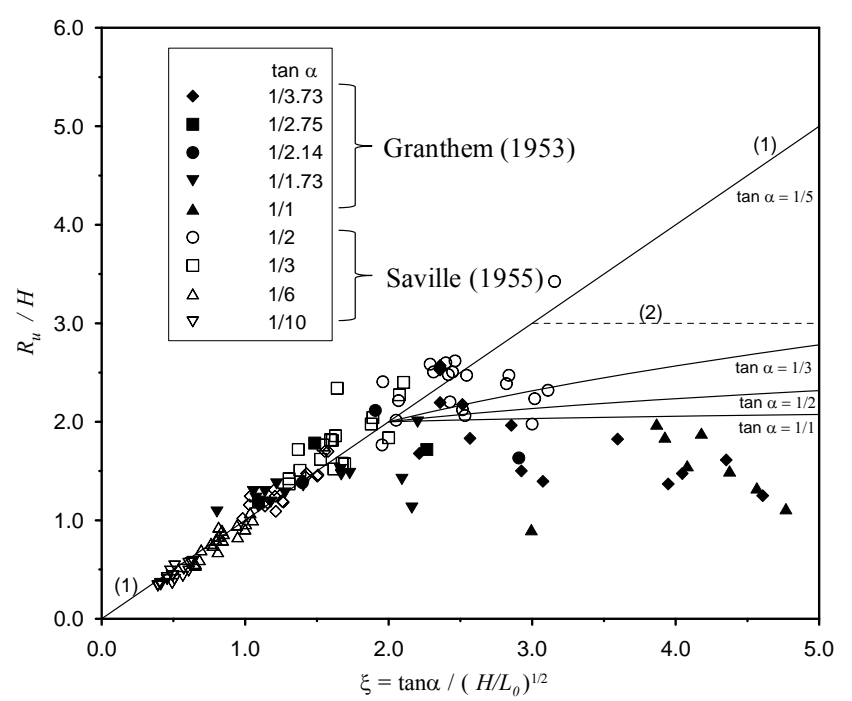

Fig. 9. Verification of empirical formulae using datasets of Granthem (1953) and Saville (1955). Line (1) is Hunt's (1959) formula and Eq. (9a); Line (2) is Hunt's (1959) formula of $R / H=3$; others are Eq. (9b). Granthem's data are given by solid markers and Saville's data are hollow markers.

Eq. (9) for $\xi \leq 3$ and $\tan \alpha \leq 1 / 2$, but are overestimated for $\xi>3$. On steeper sloping structures, the waves either travel primarily like surging breakers or the downward swash force of waves drags the rushing up water; therefore, the run-up on the slope is reduced. Accordingly, from this comparison, we conclude that Eq. (9) is valid within the range of $\xi \leq 3$ and $1 / 5 \leq \tan \alpha<1 / 2$.

In Eq. (9), it is noted that there is a different measure of wave height at the foot of toe between the experimental dataset generated by the present experiment and those of Granthem and Saville. The wave height at the structure toe was estimated by linear shoaling, but it was calculated by BE model. For a 1:1 structure slope, most of the data have much lower run-up values than estimated. As pointed out by Hughes (2004), waves rushing on this steel slope are probably surging breakers. Madsen and Fuhrman (2008) have addressed this issue recently, and proposed formula for maximum run-up and the associated flow velocity based on theoretical analysis. So their formulas are valid for a larger value of $\xi$. Their results also show that $R_{\mathrm{u}} / H$ tends to a unique curve for small $\xi$, typically following Hunt's breaking formula, whereas a family of curves for various $R_{\mathrm{u}} / H$ exists for larger $\xi$.

Figures 7 and 9 imply that the run-up heights of extreme conditions such as typhoon waves, which may have a larger Iribarren number together with a higher wave height and longer wave period, are generally overestimated by the existing formulae. By comparing Figs. 7 and 9, we can speculate that the different branch points may be a result of surging breakers or increasing downward withdraw of the fluid 
weight component, or the effect of the opposing current from the down-rush of the preceding crest, in which the wave run-up height is generally overestimated by the previous and present formulae.

The correlation coefficient and agreement index $\left(R^{2}=\right.$ 0.97 and $C_{R}=0.99$ ) between the computed results from the present numerical model and the proposed empirical equations are high. Hence, Eq. (9) can be useful for practical applications to estimate the wave run-up height for the Iribarren number whose range is beyond the conventional value of $\xi=2$ but limited to $\xi \leq 3$; moreover, the equation can also be useful for test slopes over a wide range from $1 / 10$ to $1 / 2$ for regular waves.

\section{Conclusions}

In this study, we have applied a numerical model that integrates second-order fully nonlinear BEs (Wei et al., 1995) and the analytical theory for wave breaking and run-ups (Kennedy et al., 2000) for simulating wave breaking and the subsequent run-ups for regular waves over sloping structures. Laboratory experiments were carried out in a wave flume for breaking wave run-up on $1 / 5,1 / 4$ and $1 / 3$ plane slopes. The property of breaking run-up elevation and its relationship with the Iribarren number and sloping structure were discussed.

A total of 160 numerical simulations, using with 4 sets of wave periods with 16 groups of wave steepness on 10 sloping structures, were performed. From these data, the wave run-up height for each case was collectively compared with the results of the laboratory results of regular waves. A good agreement was found, with the exception of the discrepancy for surging breakers or sloping structures larger than $1 / 1$ breaking. For a larger Iribarren number and sloping structures $(\xi>2$ and $\tan \alpha>1 / 5)$, a steeper sloping structure would produce lower wave run-up heights for a given Iribarren number. The influence of sloping structures on wave run-ups increases due to the increase in the downward swash force from the fluid weight component $\rho g \sin \alpha$. This force drags the rushing-up water on a steep slope and reduces the run-up height. A surging breaker could also produce a lower run-up (see Sect. 5).

Alternative empirical formulae are also proposed for the estimation of regular wave run-ups on different sloping structures. The new expression, Eq. (9), is valid for a wide range of the Iribarren number $\xi$ beyond the conventional range of $\xi \leq 2$ (Hunt, 1959), and for sloping structures in the range of $1 / 5<\tan \alpha<1 / 2$. In addition to the existing relationship for wave run-ups on the sloping structures of $1 / 5$, Eq. (9a), a new formula is proposed for sloping structures steeper than $1 / 5$, Eq. (9b).

A precisely defined statistical measure of Eq. (9) was examined. The tests of an agreement measure included the agreement index $C_{R}$, the mean absolute error (MAE), the root-mean-square error (RMSE), and the scattering index (SCI), respectively. The results showed that Eq. (9) offers a good prediction compared to the calculated and observed data. The details of the analysis were illustrated in Figs. 5-7. Comparisons and validations of the proposed formulae, using the previous datasets of Granthem (1953) and Saville (1955), and other empirical formulae were shown in Fig. 9.

Based on the model verification, we are confident that Eq. (9) can be applied to the modeling of wave transformations from spilling and plunging wave breaker $(\xi \leq 3)$ run-ups over a wide range of sloping structures with $1 / 5 \leq$ $\tan \alpha \leq 1 / 2$ for regular waves. The present investigation validated the model of Wei et al. (1995) for regular waves, and it serves as an important stepping stone to verify irregular waves and more complex geometries of coastal structures. In addition, our results will be very useful to establish good coastal infrastructure protection measures by, for example, delimiting buffer zones and enhancing the accuracy of coastal risk mapping.

Acknowledgements. The authors acknowledge the support from National Science Council, Taiwan, under the Grant of 97.2221E-006-261-MY3 and NSC99-3113-P-006-008. We are especially grateful to the reviewers for giving beneficial comments that were useful for improving the presentation of this paper. Statistical analysis was provided by Ping-Chang Sueh, a Ph.D. candidate of Southampton University, UK.

Edited by: S. Tinti

Reviewed by: two anonymous referees

\section{References}

Battjes, J. A.: Surf similarity, Proc. 14th Int. Conf. Coastal Engineering, ASCE, 466-480, 1974.

Fuhrman, D. R. and Madsen, P. A.: Simulation of nonlinear wave run-up with a high-order Boussinesq model, Coast. Eng., 55, 139-154, 2008.

Goda, Y.: Irregular wave deformation in the surf zone, Coast. Eng., $18,15-26,1975$.

Granthem, K. N.: A model study of wave run-up on sloping structures, Technical Report, Series 3, Issue 348, Institute of Eng. Research, Univ. of California, Berkeley, California, USA, 1953.

Hsiao, S. H., Hsu, T. W., Lin, T. C., and Chang, Y. H.: On the evolution and run-up of breaking solitary waves on a mild sloping beach, Coast. Eng., 55, 975-988, 2008.

Hsu, T. W., Chang, H. K., and Tsai, L. H.: Bragg reflection of waves by different shapes of artificial bars, China Ocean Eng., 16, 343358, 2002.

Hsu, T. W., Yang, B. D., and Tseng, I. F.: On the range of validity and accuracy of Boussinesq-type models, China Ocean Eng., 18, 93-106, 2004.

Hsu, T. W., Lan, Y. J., Wang, Y. H., and Tsai, C. Y.: Using finite element method to simulate wave transformation in the surf zone, J. Eng. Mech., ASCE, 131, 1214-1217, 2005. 
Hughes, S. A.: Estimation of wave run-up on smooth, impermeable slopes using the wave momentum flux parameter, Coast. Eng., 58, 1085-1104, 2004.

Hunt, I. A.: Design of seawalls and breakwaters. Joural of Waterway Harbors Division, ASCE, WW3, 123-152, 1959.

Kennedy, A. B., Chen, Q., Kirby, J. M., and Dalrymple, R. A.: Boussinesq modeling of wave transformation, breaking, and runup, I: 1D, J. Waterw. Port C. Ocean Eng., 126, 39-47, 2000.

Kim, G. and Lee, C.: Nwogu-type Boussinesq equations for rapidly varying topography, Asian Pacific Coasts, 118, 215-221, 2009.

Kobayashi, N.: Wave runup and overtopping on beaches and coastal structures, Adv. Coast. Ocean Eng., 5, 95-154, 1989.

Lynett, P. J., Wu, T. R., and Liu, P. F.: Modeling wave run-up with depth-intergrated equations, Coast. Eng., 49, 291-305, 2002.

Madsen, P. A. and Fuhrman, D. R.: Run-up of tsunamis and long waves in terms of surf-similarity, Coast. Eng., 55, 209-223, 2008.

Madsen, P. A., Sørensen, O. R., and Schäffer, H. A.: Surf zone dynamics simulated by a Boussinesq-type model, Part I: Model description and cross-shore motion of regular waves, Coast. Eng., 32, 255-287, 1997.

Miche, M.: Mouvements ondulatries de la mer en profondeur constante on decroissante, Annales des Ponte et Chaussees, 1, 2578, 131-164, 270-292, 369-406, 1944.

Nagayama, S.: Study on the change of wave height and energy in the surf zone, Bachelor Eng. thesis, Yokohama National University, Japan, 1983.

Nwogu, O.: Alternative form of Boussinesq equations for nearshore wave propagation, J. Waterw. Port Coastal, Ocean Eng., 119, 618-638, 1993.

Nwogu, O. and Demirbilek, Z.: Infragravity wave motions and runup over shallow fringing reefs, J. Waterw. Port Coastal, Ocean Eng., 136, 295-305, 2010.

Raubenheimer, B.: Observations and predictions of fluid velocities in the surf and swash zone, J. Geophys. Res., 107, 3190, doi:10.1029/2001JC001264, 2002.

Raubenheimer, B. and Guza, R. T.: Observations and predictions of runup, J. Geophys. Res., 101, 575-587, 1996.

Savage, R. P.: Wave run-up in roughened and permeable slopes, J. Waterw. Harbors Div., 99, 1-38, 1958.
Saville Jr., T.: Laboratory data on wave run-up and overtopping on shore structure. Technical Memorandum, 64, Beach Erosion Board, US Army Corps of Engineers, Washington, DC, USA, 1955.

Saville Jr., T.: Wave run-up on shore structures, J. Waterw. Div.ASCE, 925, 1-14, 1956.

Saville Jr., T.: Wave run-up on composite slopes, Proc. 6th Int. Conf. Coastal Engineering, ASCE, 691-699, 1958.

Shore Protection Manual: US Army Engineer Waterways Experiment Station, 4th Edn., US Government Printing Office, Washington, DC, 1984.

Stoa, P. N.: Reanalysis of wave runup on structures and beaches, U.S. Army Corps of Engineers Coastal Engineering Research Center, Ft. Belvoir, VA, TP 78-2, 1978.

Synolakis, C. E.: The run-up of long waves, Ph.D. thesis, California Institute of Technology, Pasadena, Calif., USA, 1986.

Tao, J.: Computation of wave run-up and breaking. Internal Report, Danish Hydraulics Institute, Denmark, 1983.

Tao, J.: Numerical modeling of wave runup and breaking on the beach, Acta Oceanologia Seneca Beijing, 6, 692-700, 1984.

TAW: Technical report wave run-up and wave overtopping at dikes, Technical advisory committee on flood defence, The Netherlands, 2002.

Takada, A.: On relation among wave run-up, overtopping and reflection, Proc. of JSCE, 182, 19-30, 1970 (in Japanese).

Toyoshima, O., Shuto, N., and Hashimoto, H.: Wave run-up heights on coastal dikes, -1/30-, Proc. 11th Japanese Conf. on Coastal Engineering, JSCE, 206-265, 1964 (in Japanese).

Wei, G. and Kirby, J. T.: Time-dependent numerical code for extended Boussinesq equations, J. Waterw. Port Coastal, Ocean Eng. 121, 251-263, 1995.

Wei, G., Kirby, J. T., Grilli, S. T., and Subramanya, R.: A fully nonlinear Boussinesq model for surface waves, Part 1, Highly nonlinear unsteady waves, J. Fluid Mech., 294, 71-92, 1995.

Willmott, C. J.: On the validation of models, Phys. Geogr., 2, 219232, 1981.

Zelt, J. A.: The run-up of non-breaking and breaking solitary waves, Coast. Eng., 15, 205-245, 1991. 